\title{
An inexpensive method of indirect ophthalmoscopy
}

\section{K R BISHAI}

From the Department of Ophthalmology, London Hospital, Whitechapel, London E1 1BB

SUMMARY Attention is drawn to the possibility of performing monocular indirect ophthalmoscopy with a pen torch and a condensing lens when facilities for binocular indirect ophthalmoscopy are not available.

Indirect ophthalmoscopy is most commonly performed with a head- or spectacles-mounted source of illumination positioned in the midline of the forehead. Both hands are thus free to hold a condensing lens and to manipulate the lids or a scleral depressor or to make a retinal drawing. In addition the mounted viewing system optically narrows the interpupillary distance of the observer to provide a stereoscopic view of the fundus image.

However, such advantages may not be necessary when the examiner merely requires a wide angle view of the posterior fundus and the equator. In such cases

Correspondence to Mr K R Bishai, FRCS Ed. a hand held bright torch and a condensing lens can constitute a readily available system for indirect monocular ophthalmoscopy.

\section{Material and methods}

The examiner holds a pen torch with one hand just above or below the fixating eye, resting the hand against the forehead or cheek bones to maintain steadiness, and projects the light on to the patient's dilated pupil (Fig. 1). The red glow of the fundus will be observed in the pupillary plane. By intercepting the fundus reflex with a condensing lens of appropriate power-for example, aspheric $+20 \mathrm{D}$ or +28

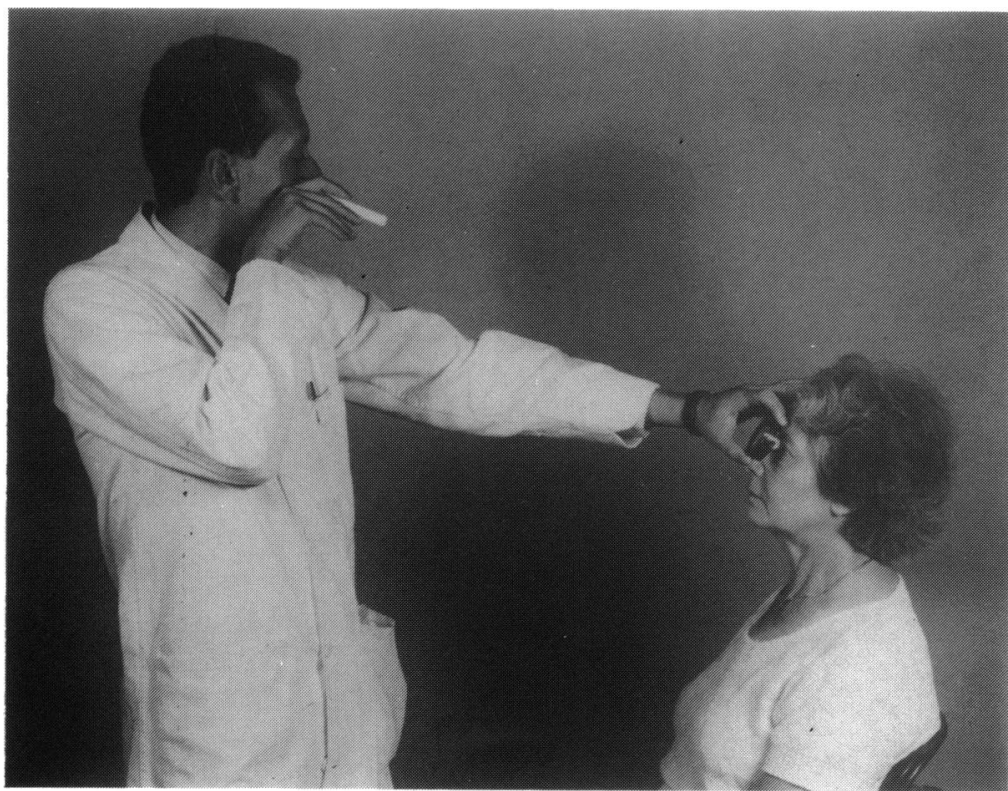

Fig. 1 Examination of patient by indirect monocular ophthalmoscopy. 
D-a real and inverted image of the fundus will form between the examiner and the patient.

\section{Discussion}

The principle of observing the fundus by separating the reflected image from the illuminated beam was first discovered by Purkinje in 1823 and rediscovered independently by Cumming in 1846 and Brucke in $1847 .{ }^{1}$ Indirect ophthalmoscopy owes its development to the works of Nagel in 1846, Haab in 1910, and Gut in $1948 .{ }^{2}$ Although a number of monocular indirect ophthalmoscopes are still obtainable, its practice has been largely eclipsed by the superior imaging system of the modern binocular indirect ophthalmoscopy. ${ }^{3}$

However, equipment for binocular indirect ophthalmoscopy is cumbersome and not readily available outside ophthalmic departments. Modern pen torches provide a sufficiently powerful source of illumination, enabling ophthalmologists to perform indirect ophthalmoscopy in the absence of better equipment. The emergent monocular image is of adequate quality and will outline gross fundus pathology posterior to the equator. The examination can be performed easily on medical wards or in remote areas where convenient facilities may not be available or serviceable.

I am grateful to Mr I D Berle and other members of the Photographic Department of the London Hospital for their assistance.

\section{References}

1 Reese PD. The neglect of Purkinje's technique of ophthalmoscopy prior to Helmhotz's invention of the ophthalmoscope. Ophthalmology 1986; 93: 1457-60.

2 Duke-Elder S. System of ophthalmology. London: Kimpton, 1962: 7: 303.

3 Rubin ML. The optics of indirect ophthalmoscopy. Surv Ophthalmol 1964; 9: 449-64.

Accepted for publication 8 February 1988. 